\title{
Sensor Fusion Algorithm Based on Extended Kalman Filter for Estimation of Ground Vehicle Dynamics
}

\author{
Daniel Barbosa ${ }^{\dagger}$ António Lopes*† and Rui Esteves Araújo*† \\ *INESC TEC \\ ${ }^{\dagger}$ Faculty of Engineering, University of Porto, 4200-465 Porto, PORTUGAL \\ Email:\{daniel.barbosa, antonio.lopes, raraujo\}@fe.up.pt
}

\begin{abstract}
The current vehicle stability control techniques relies on an accurate sensor information and a complete system definition, such information is not easily obtained and requires expensive sensor technology. In this work it is presented a fusion algorithm for estimating the vehicle handling dynamic states, using inertial measurements combined with Global Positioning System (GPS) information, based on the Extended Kalman Filter algorithm (EKF). The proposed method will be able to track the state of the variable vector that includes the yaw rate, lateral velocity and longitudinal velocity of the vehicle using the information of the available sensors combined with the nonlinear model of the system. In order to validate the proposed sensor fusion algorithm a simulation with a high-fidelity CarSim model is carried out and its sensors are compared with Extended Kalman Filter state variables.
\end{abstract}

\section{INTRODUCTION}

In recent years most ground vehicles have been equipped with Advanced Driver Assistance Systems (ADAS) to improve driver and passenger security. There are a lot of control systems such as Anti-lock Brake System (ABS), Electronic Stability Control (ESC), Rollover Prevention System, and so on. These systems have proven to be helpful in reducing vehicle crashes and will also be very useful for overactuated electric vehicles (EVs) in the future [1]. However, the performance of the ADAS depends on the accuracy of the information regarding the longitudinal and lateral velocities, other state variables and normal tyre forces, which can be difficult to be measured in a cost-efficient way. Although optical sensors could be used to measure directly the vehicle lateral velocity, there are practical issues such as accuracy, cost and reliability that inhibit production vehicles from using it. Therefore, the estimation of vehicle longitudinal and lateral velocities and lateral forces based on other vehicle inputs/outputs is critical and has been widely investigated in literature.

Baffet et al. in [2] explore the use of two decoupled observers - in a first stage, a sliding-mode observer is used to calculate tire/road forces, and an EKF is used in a second stage to estimate the sideslip angle and cornering stiffness. It was used a tire-force model to implement the EKF, achieving good estimation of the sideslip angle. Kim in [3] presents a procedure for off-line identification of lateral tyre forces, using a simple vehicle model, an EKF and experimental road data, achieving a good estimation of lateral tyre forces.
Chen and Hsieh in [4] develop a sideslip angle estimator based on a kinematic model. It is discussed the performance of this system in a tire/road parameters and uncertainties dependent model, which rapidly deteriorates with different road conditions.

Currently, we are on the verge of a new paradigm shift: the virtual sensing. This concept consists on combination of computational models, parameters and data from physical sensors to provide more reliable estimations of the quantities of interest. Dakhlallah et al. in [5] use an EKF to estimate the tire/road forces alongside a friction model to evaluate the sideslip angle. It achieves a good estimation and concludes that it is possible to obtain a comparable response to the expensive sensors currently used to measure the sideslip angle. This shift towards virtual sensing has a large potential to improve the performance of motion controllers in overactuated EVs. This idea is based essentially on sensor fusion that is an effective way to satisfy the increasing demand on system performance, fault tolerance and reliability of the motion controllers for EVs. Typically the sensors used in a vehicle perform a single specific task and do not interact strongly with other sensors. Basically it is only an input to controller. Sensor fusion is the combination of information given by multiple sensors, obtaining superior information to the one the sensors give individually. On other hand, when some quantity cannot be measured directly, it is often necessary to estimate it using the measurements that are available. It should be added that in emergency situations, sideslip is necessary to detect a sliding or skidding vehicle, which may have normal yaw rates. Also in these situations, the longitudinal velocity cannot be accurately measured by wheel speed because of excessive wheel slip. Thanks to the sensor fusion flexibility the information gathered in this way can help drivers and motion controllers make better judgments, resulting in smoother and safer driving. Daily and Bevly in [6] explore the use of GPS to calculate the sideslip angle of a vehicle, in order to control its stability, pointing out the possibility of developing higher performance controllers combining the GPS with an Inertial Measurement Unit (IMU). It was also presented a method which calculates and predicts the lateral forces of the tires. Morrison and Cebon in [7] use a vehicle model and a nonlinear tire model alongside a nonlinear Kalman Filter to estimate the sideslip of articulated heavy 
vehicles in low friction conditions.

The concept of sensor fusion is not new. The emergence of new sensors, advanced processing techniques, and improved processing hardware have made real-time fusion of information increasingly viable for automotive applications. Algorithms implementing that fusion system for EVs are a very promising field of research, and towards this direction this paper aims to contribute. There is a broad range of classification for sensor fusion and while there is no norm, Khaleghi et al. in [8] and Castanedo in [9] suggest a succinct and extensive classification of the algorithms and of the data that it fuses, based on different metrics. This paper will focus in complementary and centralized fusion; the former due to the different kind of sensor input to be fused and the latter because a single processor will receive the information directly from the sensors. The Kalman Filter method has been proved to be the most powerful tool for multi-sensor data fusion problems at a low computational load. The algorithm combines the available measurements from the GPS and IMU with dynamic model to enhance the estimation of key vehicle dynamic states. Design goals include a reduction of computational complexity compared to the observers in order to make the Kalman filter suitable for implementation in embedded hardware. The design is based on a standard sensor configuration, and is subjected to extensive testing in realistic conditions with CarSim Software. It is shown that a set of two Kalman filters can provide an accurate estimation of the main state variables for motion control. The filter estimates can be used to implement control algorithms, as Fig. 1 illustrates. The paper is organized as follows: section II introduces the Kalman Filter algorithm that accomplishes the sensor fusion, section III demonstrates the performance of the algorithm and section IV presents a discussion of what was accomplished.

\section{SEnsor Fusion Algorithm}

In this section it will be presented the sensor fusion algorithm proposed for a ground vehicle. First it will be introduced the mathematical model of the system used to implement the Kalman algorithm, followed by a coordinate transformation to obtain the sensors information in the desired coordinate frame, and finally the design and implementation of the EKF algorithm. In Fig. 1 is presented an overview of the proposed sensor fusion structure.

\section{A. Vehicle Model}

The laws of motion of a rigid body are expressed by two key motions, the translation motion and the rotation motion. The definition of this equations are based on the Newton-Euler laws of motion that express the behaviour of a rigid body as presented by (1) [10].

$$
\begin{aligned}
\dot{v}_{x} & =\frac{1}{m}\left(F_{x}\right)+v_{y} \dot{\psi} \\
\dot{v}_{y} & =\frac{1}{m}\left(F_{y}\right)-v_{x} \dot{\psi} \\
\ddot{\psi} & =\frac{M_{z}}{I_{z}}
\end{aligned}
$$

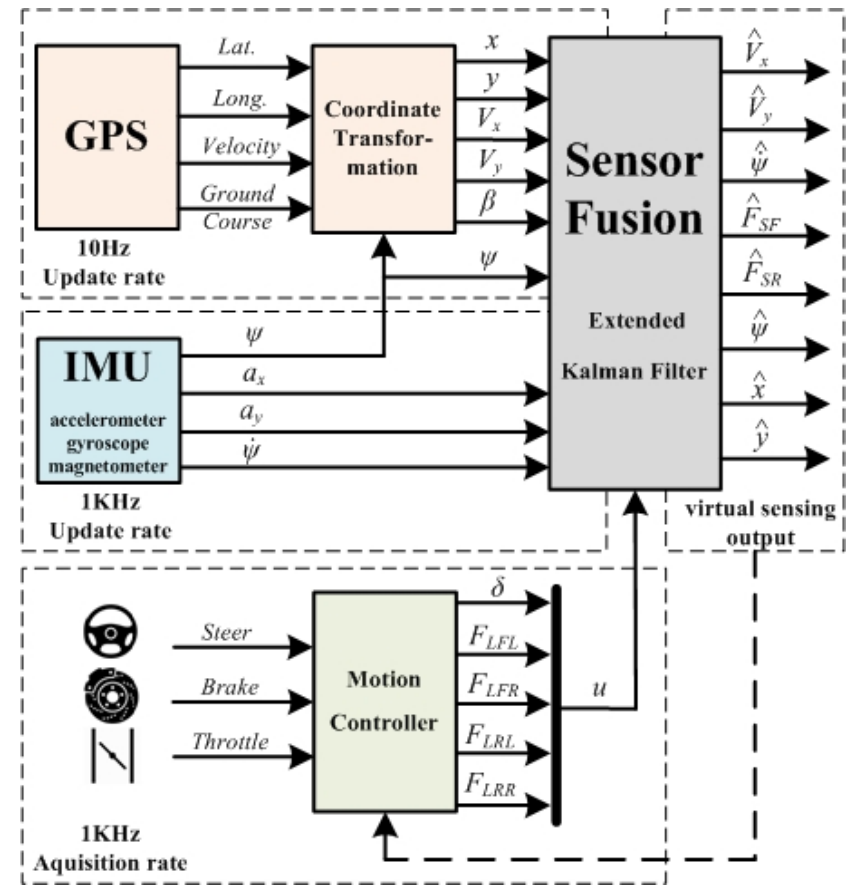

Fig. 1. Outline of the proposed sensor fusion system

Where $m$ express the total mass of the vehicle, $I_{z}$ the inertial moment of the vehicle in the vertical axis and $M_{z}$ the total moment generated by the forces applied in each individual wheel presented in (2) [11].

$$
\begin{aligned}
M_{z}= & \left(\left(F_{S F L}-F_{S F R}\right) \sin (\delta)+F_{L R R}-F_{L R L}+\left(F_{L F R}\right.\right. \\
& \left.\left.-F_{L F L}\right) \cos (\delta)\right) \frac{l_{s}}{2}-\left(F_{S R R}+F_{S R L}\right) l_{r}+\left(\left(F_{S F R}\right.\right. \\
& \left.\left.+F_{S F L}\right) \cos (\delta)-\left(F_{L F R}+F_{L F L}\right) \sin (\delta)\right) l_{f}
\end{aligned}
$$

The state variables of the system are the vehicle's longitudinal velocity $v_{x}$, the lateral velocity $v_{y}$ and the yaw rate $\dot{\psi}$. The forces $F_{x}$ and $F_{y}$ represent the sum of all longitudinal and lateral forces, respectively, applied to the vehicle and can be defined as (3) and are explicitly presented in Fig. 2.

$$
\left\{\begin{array}{rr}
F_{x}= & \left(\left(F_{L F L}+F_{L F R}\right) \cos (\delta)+\left(F_{L R L}+F_{L R R}\right)\right. \\
& \left.-\left(F_{S F L}+F_{S F R}\right) \sin (\delta)\right) \\
F_{y}= & \left(\left(F_{L F L}+F_{L F R}\right) \sin (\delta)+\left(F_{S R L}+F_{S R R}\right)\right. \\
+ & \left.\left(F_{S F L}+F_{S F R}\right) \cos (\delta)\right)
\end{array}\right.
$$

Where $F_{S j w}$ and $F_{L j w}$ represent the forces in the lateral and longitudinal axis in the $j w$ wheel respectively.

In the present formulation it will be considered that the difference between the lateral forces of the front and rear axis are negligible resulting in $F_{S F}=F_{S R}$ and it is defined $F_{S F}$ and $F_{S R}$ as the lateral forces applied in the front and rear axis respectively as presented in (4).

$$
F_{S F}=F_{S F L}+F_{S F R}, \quad F_{S R}=F_{S R L}+F_{S R R}
$$



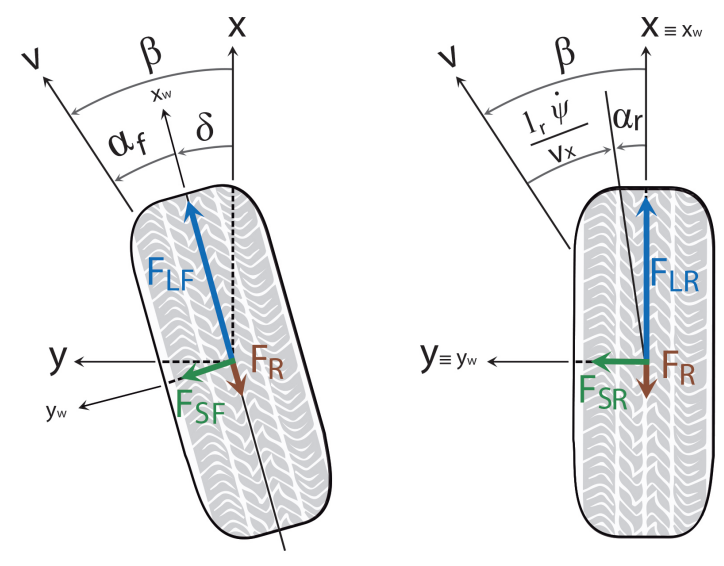

Fig. 2. Forces applied to the front(left) and rear(right) wheel [10]

It will also be considered the drag force $\left(F_{D R A G}\right)$ dynamic as presented in (5) [11]:

$$
F_{D R A G}=\frac{1}{2} \rho C_{D} A v^{2}
$$

where $\rho$ is the atmospheric air density, $C_{D}$ the drag factor, $A$ the area of the vehicle frontal projection and $v$ the total speed of the vehicle.

The adopted non-linear model is presented in (6).

$$
\begin{aligned}
\dot{v}_{x}= & \frac{1}{m}\left(\left(F_{L F L}+F_{L F R}\right) \cos (\delta)+\left(F_{L R L}+F_{L R R}\right)\right. \\
& \left.-\left(F_{S F}\right) \sin (\delta)-F_{D R A G}\right)+v_{y} \dot{\psi} \\
\dot{v}_{y}= & \frac{1}{m}\left(\left(F_{L F L}+F_{L F R}\right) \sin (\delta)+\left(F_{S R}\right)\right. \\
& \left.+\left(F_{S F}\right) \cos (\delta)\right)-v_{x} \dot{\psi} \\
\ddot{\psi}= & \frac{1}{I_{z}}\left(\left(F_{L R R}-F_{L R L}+\left(F_{L F R}-F_{L F L}\right) \cos (\delta)\right) \frac{l_{s}}{2}\right. \\
& +\left(\left(F_{S F}\right) \cos (\delta)-\left(F_{L F L}+F_{L F R}\right) \sin (\delta)\right) l_{f} \\
& \left.-\left(F_{S R}\right) l_{r}\right)
\end{aligned}
$$

Where $l_{s}, l_{f}, l_{r}$ are the length of the axis, the distance between the front axis and the center of gravity (CoG), and the distance between the rear axis and the CoG.

\section{B. Coordinate Transformation}

The GPS is placed with the IMU in the CoG of the vehicle. The GPS presents the position of the system in a global geodetic frame, the Geodetic Coordinate System (GCS), while the IMU is aligned with the local navigation frame, that is associated with the vehicle's CoG [12]. The velocity and ground course $(G C)$ from the GPS are also obtained in the navigation frame. In order to convert the GPS readings to a 2D Cartesian coordinate system, it will be considered an equirectangular projection where latitude lines are straight, parallel and equally spaced between each other, and so are the meridians of longitude [13]. This is achieved with a scaling factor applied to the longitude readings. This method greatly diminishes the computational burden when compared to the typical 3D geodetic-Cartesian conversion, as it assumes $y=R \varphi$ and $x=R \lambda \cos \left(\varphi_{0}\right)$, where $R$ is the Earth's radius, $\varphi$ is the latitude, $\lambda$ is the longitude, $\varphi_{0}$ is the origin measurement's latitude and $\cos \left(\varphi_{0}\right)$ the scale factor that makes this calculation correct for the respective pair of equidistant latitude lines above and below the equator line. To obtain distance between one point and the starting point in meters, we do $\sqrt{\left(x-x_{0}\right)^{2}+\left(y-y_{0}\right)^{2}}$, where $x_{0}$ and $y_{0}$ are the origin latitude and longitude that are set when the algorithm starts.

The available sensor information will enable the access to three angles: the heading $(\psi)$ that gives the physical orientation of the vehicle, the $G C$ of the GPS that gives the orientation of the movement of the vehicle, and the sideslip angle $(\beta)$ given by (7).

$$
\beta=\psi-G C
$$

Notice also that the longitudinal and lateral velocity can be related through the sideslip angle as expressed in (8).

$$
v_{x}=v \cos (\beta), v_{y}=v \sin (\beta)
$$

The longitudinal and lateral velocity of the $\mathrm{CoG}$ of the vehicle can be referred to the origin frame by applying the rotation matrix with a $\psi$ rotation angle as presented in (9a) and in (9b).

$$
\begin{aligned}
& \dot{x}=v_{x} \cos (\psi)-v_{y} \sin (\psi) \\
& \dot{y}=v_{x} \sin (\psi)+v_{y} \cos (\psi)
\end{aligned}
$$

\section{Kalman Filter Design}

The Extended Kalman Filter is a non-linear observer that aims to minimize the covariance error of the system state estimation. The Kalman observer is especially useful when the noise of the system measure or process is a white noise with zero mean [3], [14].

The introduction of a non-linear observer employs the nonlinear vehicle model presented in (6) combined with the sensor dynamic presented in (9) and linearize it in each computational step. The continuous-time non-linear system can be defined in the general form as [3]:

$$
\begin{aligned}
& \dot{x}(t)=f(t, x(t), u(t))+w(t) ; \\
& y(t)=h(t, x(t), u(t))+\gamma(t) ;
\end{aligned}
$$

where $w(t) \sim N(0, Q(t))$ and $\gamma(t) \sim N(0, R(t))$ represent the process noise (disturbance) and the measurement noise respectively and are assumed to be zero mean Gaussian noise [14].

It is assumed from now on the discrete system of (10), by assuming the Forward Euler approximation based on the truncated Taylor series expansion presented in (11):

$$
X_{k+1}=X_{k}+T_{s} f\left(X_{k}, U_{k}\right)
$$


where $T_{s}$ is the computational step $\left(t_{k+1}=t_{k}+T_{s}\right)$.

The discrete state vectors are $X_{k}$ and $Y_{k}$ are defined as:

$$
\begin{aligned}
X_{k} & =\left[v_{x}, v_{y}, \dot{\psi}, F_{S F}, F_{S R}, \psi, x, y\right]^{T} \\
Y_{k} & =\left[v_{x}, v_{y}, \dot{v}_{x}, \dot{v}_{y}, \psi, \dot{\psi}, \beta, x, y\right]^{T}
\end{aligned}
$$

The measurement of the accelerations is available as well as the yaw rate and heading reference of the system through the IMU. The longitudinal velocity, position and sideslip angle of the system are obtained through GPS. The longitudinal forces applied on each wheel of the vehicle are assumed to be the control variables that are available. The steering angle $(\delta)$ is also available and is assumed as part of the control variable, $U_{k}$ as it can be seen in Fig. 1 and is expressed by:

$$
U_{k}=\left[F_{L F L}, F_{L F R}, F_{L R L}, F_{L R R}, \delta\right]^{T}
$$

The intrinsic difference between the available sensors poses an important problem regarding the sample rate of each sensor and the computational time of the proposed sensor fusion algorithm. Typically, GPS acquires new samples at a slower rate $(5-10 \mathrm{~Hz})$ than the IMU, which has a much higher sample frequency $(1 k H z)$.

In order to cope with this limitation of the GPS, it is proposed a method that allows the EKF algorithm to update at a higher frequency using just the IMU measures, introducing the GPS measure to correct the system variables [15]. As a result, the EKF is updated at the same frequency as the IMU $(1 \mathrm{kHz})$ and correct the state variables with the information provided by the GPS at $10 \mathrm{~Hz}$. The only exception is the magnetometer that is included in the IMU, since it has a slower acquisition rate on par with the GPS $(10 \mathrm{~Hz})$ and will be processed alongside the GPS data, despite coming from the IMU. The information of all available sensors is acquired at the same time in order to synchronize the sensor data with the algorithm.

The design of this method implies that the algorithm will be defined in two distinct scenarios: the one where only the IMU information is available, and other where all the sensor information is available. The first scenario will be executed at $1 \mathrm{kHz}$ and the second scenario will replace the previous scenario, once the GPS measure is available. If the GPS fails temporarily, the first scenario will be executed continuously until there's a new GPS reading.

The non-linear model $f\left(X_{k}, U_{k}\right)$ can be expressed as function of the state and control variables, based on the equations presented in (6) and the positioning dynamic evolution presented previously. The dynamic of the state variables $F_{S F}$ and $F_{S R}$ are defined as unknown in this model.

The measured output of the sensors, that are positioned in the vehicle's CoG, are expressed by the function $h\left(X_{k}, U_{k}\right)$. This function will be defined differently in the two scenarios, as the velocity, ground course and position provided by the GPS and the heading provided by the magnetometer will not be available.

In the EKF formulation, the system is linearized in each computational step through the Jacobian matrix $F_{k}$ and $H_{k}$ of the non-linear model.

$$
F_{k}=\frac{\partial f\left(X_{k}, U_{k}\right)}{\partial X_{k}}, \quad H_{k}=\frac{\partial h\left(X_{k}, U_{k}\right)}{\partial X_{k}}
$$

The Kalman filter algorithm relies on the prediction of the state variable which is obtained through the Jacobian matrix. The Kalman filter is a recursive algorithm, which means that the prediction of the current state is obtained through the previous estimation. There are two main steps in the Kalman algorithm: the prediction and the update [16].

The prediction of the state variables are obtained through the non linear equations of the model. The prediction of the covariance matrix $P_{k}$ is also performed through (15) and express the accuracy of the state estimation [16].

$$
P_{k}=F_{k} P_{k-1} F_{k}^{T}+Q_{k}
$$

In the update process it is computed the optimal Kalman gain through the Riccati equation, presented in (16). The state variable is also updated with a component generated by the Kalman gain and the measured residual, presented in (17). Finally, the estimate covariance matrix $P_{k}$ is also updated [16], presented in (18).

$$
\begin{aligned}
K_{k} & =\frac{P_{k} H_{k}^{T}}{H_{k} P_{k} H_{k}^{T}+R_{k}} \\
X_{k} & =X_{k-1}+K_{k}\left(Y_{k}-H_{k} X_{k}\right) \\
P_{k} & =\left(I-K_{k} H_{k}\right) P_{k}
\end{aligned}
$$

It is important to notice that, despite having two different scenarios, the dimensions of the state and process matrices does not change. The prediction and update equations for each situation are the same. The changes of the algorithm are only present in the definition of the measurement vector $h\left(X_{k}, U_{k}\right)$, the inherent Jacobian matrix $H_{k}$ and in the definition of the covariances matrices $Q_{k}$ and $R_{k}$ that are configured differently. As the measurement of the velocity, position, heading and sideslip angle are no longer present, the covariances matrices are reconfigured in order to impose a greater dependency on the model.

\section{RESULTS}

The sensor fusion algorithm was tested with maneuvers produced by the commercial software CarSim. The maneuvers consist of a Double Line Change (DLC) at $60 \mathrm{Km} / \mathrm{h}, 90 \mathrm{~km} / \mathrm{h}$, $120 \mathrm{~km} / \mathrm{h}$ and $160 \mathrm{~km} / \mathrm{h}$ and a J-turn at $60 \mathrm{~km} / \mathrm{h}, 90 \mathrm{~km} / \mathrm{h}$ and $120 \mathrm{~km} / \mathrm{h}$. The procedures are carried out in a simulation environment and the maneuver presented is defined by the driver model present in the CarSim software. As a result, the inputs may vary slightly at different velocities.

The validation of the proposed sensor fusion algorithm, implemented in Matlab, is defined by recovering the driver inputs from the CarSim in different scenarios and comparing the resulting signals obtained with the measured variables from Carsim simulation tool. In order to evaluate the performance of the proposed EKF it is introduced a Gaussian noise to each measurement with a standard deviation value equal to the typical value for low-grade sensors, these values are presented in table I. 


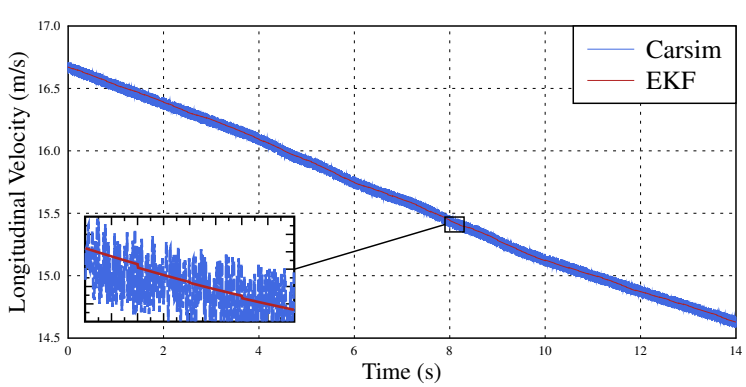

(a)

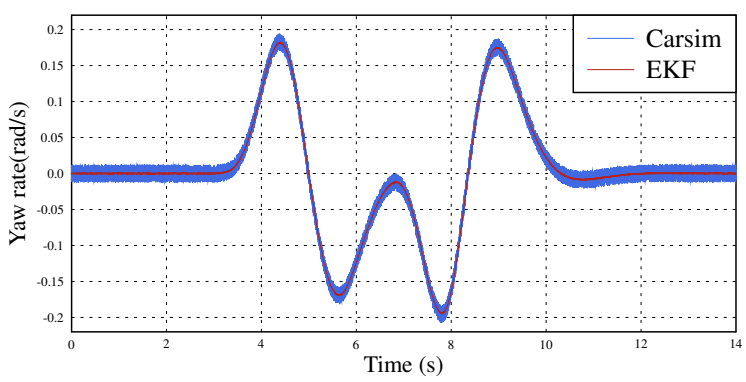

(c)

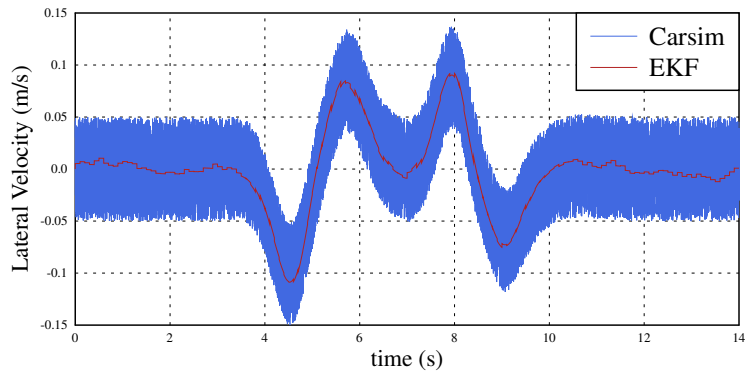

(b)

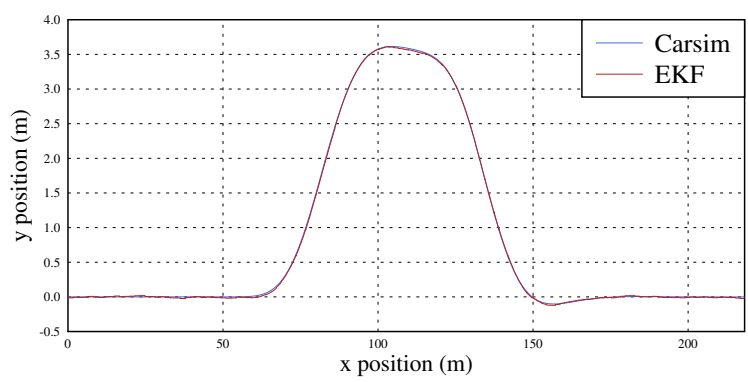

(d)

Fig. 3. DLC maneuver at $60 \mathrm{~km} / \mathrm{h}$ (a) Longitudinal velocity (b) Lateral velocity (c) Yaw Rate (d) Position

TABLE I

Standard Deviation of SEnsor Measurements

\begin{tabular}{|c|c|ll|}
\hline \multirow{2}{*}{ GPS } & \multicolumn{2}{|c|}{ Standard Deviation } \\
\hline & Velocity & 0.1 & $(\mathrm{~m} / \mathrm{s})$ \\
\cline { 2 - 4 } & Ground Course & 0.01 & $(\mathrm{rad})$ \\
\cline { 2 - 4 } & Position & 0.1 & $(\mathrm{~m})$ \\
\hline \multirow{3}{*}{ IMU } & Acceleration & 0.025 & $\left(\mathrm{~m} / \mathrm{s}^{2}\right)$ \\
\cline { 2 - 4 } & Yaw Rate & 0.002 & $(\mathrm{rad} / \mathrm{s})$ \\
\cline { 2 - 4 } & Heading & 0.01 & $(\mathrm{rad})$ \\
\hline
\end{tabular}

The results of the state variables of the DLC maneuver at $60 \mathrm{Km} / \mathrm{h}$ are presented in Fig. 3. The dynamic of the longitudinal velocity of the vehicle is presented in Fig. 3a and it is compared with the result of the sensor fusion algorithm. The lower sample rate of the longitudinal velocity hinders the EKF estimation as it only relies on the proposed model to estimate the new value of $v_{x}$, but despite this it achieves a good estimation. The longitudinal velocity estimation uses the model to update the sate variable value and uses the measurement to correct its value at a lower rate, as it can be seen in Fig. 3a. One of the most challenging state information to obtain is the lateral velocity due to the non-linear characteristic of the lateral force generated in each tire and the difficulty of modelling this forces in a simple and effective way. Fig. $3 \mathrm{~b}$ shows the performance of the proposed method on estimating the lateral velocity of the vehicle. The model's lack of knowledge of the lateral force dynamic creates a difficulty in the estimation of the lateral velocity. Nonetheless, the sensor fusion algorithm is still capable to obtain a fair estimation of the state variable from the lateral acceleration information.
The yaw rate estimation is present in Fig. 3c, where a good performance of the algorithm was achieved. The position, despite not being the main focus of this paper, is also being estimated, as shown in Fig. 3d.

The comparisons between the measured and estimated value of the DLC maneuver at different velocities are presented in Fig. 4a and in Fig. 4c, in which the error of the lateral velocity and the yaw rate of the vehicle are shown. The error in the lateral velocity increases as the dynamic of the maneuver increases, which is expected as we are dealing with higher lateral forces and therefore the estimation/prediction becomes harder. The yaw rate estimation presents an interesting result for every maneuver due to the direct measurement of the variable with high sample rate combined with a good dynamic model and sensor redundancy. It is worth noticing that the maneuver finishes at different time instants as the velocity in each procedure increases.

In Fig. 4b and Fig. 4d we have the equivalent analysis made for the a different type of maneuver, the J-turn, at three different speeds. The error of the lateral velocity increases with velocity due to a greater dynamic of the maneuver, as in the DLC, and the yaw rate estimation also presents an interesting result, having a peak when the maneuver starts but managing to stabilize as the maneuver continues.

\section{CONCLUSION}

It was proposed a sensor fusion algorithm capable of tracking the yaw rate, lateral velocity and longitudinal velocity of the vehicle at high frequency, managing to also track the position of the vehicle. This was achieved with a non-linear 


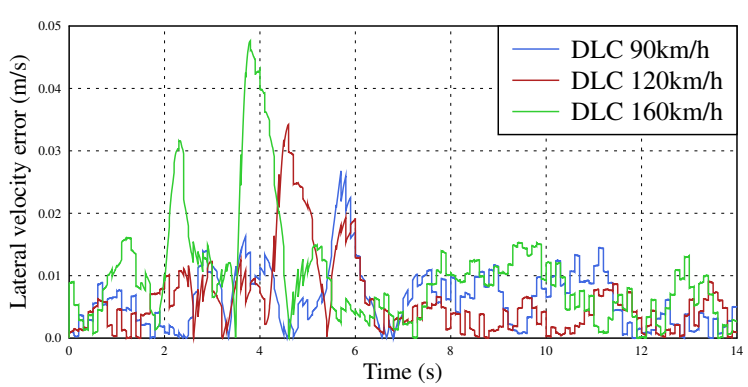

(a)

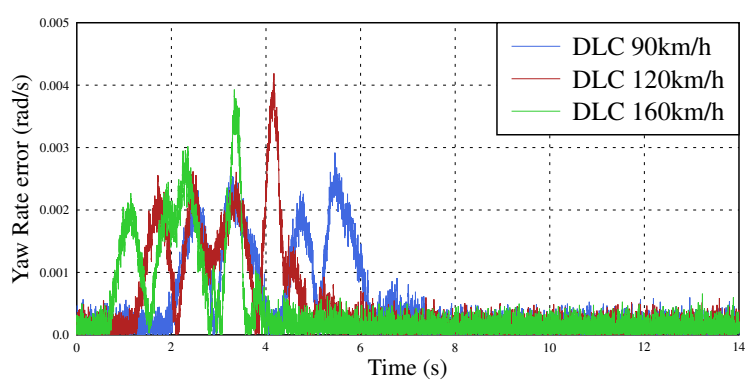

(c)

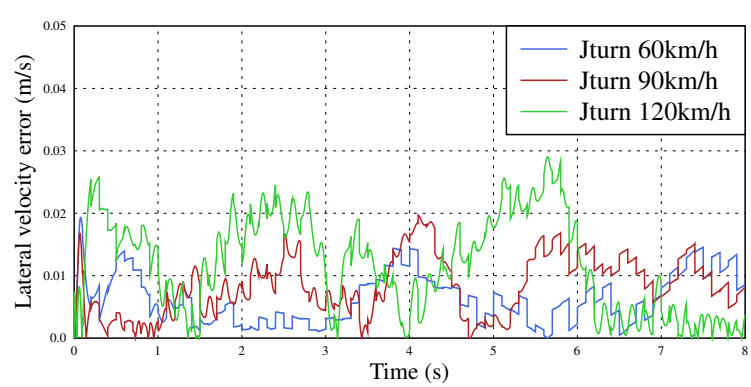

(b)

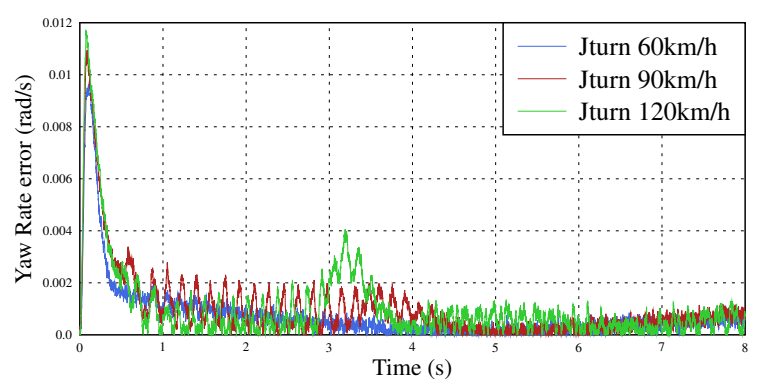

(d)

Fig. 4. Error of the sate variables: (a) Lateral velocity in DLC maneuver (b) Lateral velocity in J-turn maneuver (c) Yaw rate in DLC maneuver (d) Yaw rate in J-turn maneuver

model that describes the vehicle's dynamics, using low-grade inertial and GPS sensors. This algorithm uses two EKF in order to integrate both sensors, which allows the algorithm to estimate the vehicle's velocity with a slower acquisition of the velocity, compared to the acceleration and yaw rate. The validation is implemented by comparing the estimated results with those simulated by the CarSim and it is shown a promising tracking performance with a good convergence speed and stability. It is also shown the ability of the algorithm to work without GPS information and its capability to correct the drift from the IMU when it has GPS measurements available, solving individual limitations of both sensors.

Our future work would cover a practical application of the algorithm presented here.

\section{ACKNOWLEDGMENT}

The authors want to express gratitude for the financial support by the Electrotechnical and Computer Engineering Department of Faculty of Engineering of the University of Porto.

\section{REFERENCES}

[1] N. Mutoh and Y. Nakano, "Dynamics of front-and-rear-wheelindependent-drive-type electric vehicles at the time of failure," Industrial Electronics, IEEE Transactions on, vol. 59, no. 3, pp. 1488-1499, 2012.

[2] G. Baffet, A. Charara, and D. Lechner, "Experimental evaluation of a sliding mode observer for tire-road forces and an extended kalman filter for vehicle sideslip angle," in Decision and Control, 2007 46th IEEE Conference on. IEEE, 2007, pp. 3877-3882.

[3] J. Kim, "Identification of lateral tyre force dynamics using an extended kalman filter from experimental road test data," Control Engineering Practice, vol. 17, no. 3, pp. 357-367, 2009.
[4] B.-C. Chen and F.-C. Hsieh, "Sideslip angle estimation using extended kalman filter," Vehicle System Dynamics, vol. 46, no. S1, pp. 353-364, 2008.

[5] J. Dakhlallah, S. Glaser, S. Mammar, and Y. Sebsadji, "Tire-road forces estimation using extended kalman filter and sideslip angle evaluation," in American Control Conference, 2008. IEEE, 2008, pp. 4597-4602.

[6] R. Daily and D. M. Bevly, "The use of gps for vehicle stability control systems," Industrial Electronics, IEEE Transactions on, vol. 51, no. 2, pp. 270-277, 2004.

[7] G. Morrison and D. Cebon, "Sideslip estimation for articulated heavy vehicles in low friction conditions," in 2015 IEEE Intelligent Vehicles Symposium (IV). IEEE, 2015, pp. 65-70.

[8] B. Khaleghi, A. Khamis, F. O. Karray, and S. N. Razavi, "Multisensor data fusion: A review of the state-of-the-art," Information Fusion, vol. 14, no. 1, pp. 28-44, 2013.

[9] F. Castanedo, "A review of data fusion techniques," The Scientific World Journal, vol. 2013, p. 19, 2013. [Online]. Available: http://dx.doi.org/10.1155/2013/704504

[10] R. N. Jazar, Vehicle dynamics: theory and application. Springer Science \& Business Media, 2013.

[11] U. Kiencke and L. Nielsen, "Automotive control systems: For engine, driveline, and vehicle," Measurement Science and Technology, vol. 11, no. 12, p. 1828, 2000. [Online]. Available: http://stacks.iop.org/0957$0233 / 11 / \mathrm{i}=12 / \mathrm{a}=708$

[12] R. Christensen, N. Fogh, A. la Cour-Harbo, and M. Bisgaard, "Inertial navigation system," Department of Control Engineering, Aalborg University, 2008.

[13] J. P. Snyder, Flattening the earth: two thousand years of map projections. University of Chicago Press, 1997.

[14] M. C. Best, T. Gordon, and P. Dixon, "An extended adaptive kalman filter for real-time state estimation of vehicle handling dynamics," Vehicle System Dynamics, vol. 34, no. 1, pp. 57-75, 2000.

[15] M. Tailanián, S. Paternain, R. Rosa, and R. Canetti, "Design and implementation of sensor data fusion for an autonomous quadrotor," in 2014 IEEE International Instrumentation and Measurement Technology Conference (I2MTC) Proceedings. IEEE, 2014, pp. 1431-1436.

[16] T. A. Wenzel, K. Burnham, M. Blundell, and R. Williams, "Dual extended kalman filter for vehicle state and parameter estimation," Vehicle System Dynamics, vol. 44, no. 2, pp. 153-171, 2006. 